\title{
The pro-survival Bcl-2 family member A1 delays spontaneous and FAS ligand-induced apoptosis of activated neutrophils
}

\author{
Robyn L. Schenk ${ }^{1,2,5}$, Lahiru Gangoda ${ }^{1,2}$, Kate E. Lawlor ${ }^{3,4}$, Lorraine A. O'Reilly ${ }^{1,2}$, Andreas Strasser ${ }^{1,2}$ and \\ Marco J. Herold (iD ${ }^{1,2}$
}

Neutrophils have a short lifespan that is extended after exposure to granulocyte macrophage colony stimulating factor (GM-CSF) or lipopolysaccharide (LPS) ${ }^{1}$. While the survival is regulated by BCL-2 family proteins ${ }^{2}$, it is not known which pro-survival proteins are involved. GM-CSF stimulation in neutrophils upregulates A1, but A1-deficient mice showed no defects in this cell type ${ }^{3}$. MCL-1 is critical for the survival of quiescent neutrophils ${ }^{4,5}$, but it is not known whether the same holds true after activation. We hypothesized that A1 and MCL-1 have overlapping roles in the survival of activated neutrophils.

We generated mutant mice deficient for $\mathrm{A} 1$ and lacking

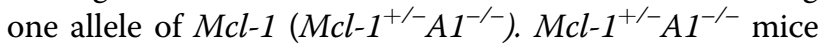
are grossly normal in the haematopoietic compartment, with only a small reduction in lymphocyte numbers, similar to ${\mathrm{Mcl}-1^{+/-}}$mice $^{6}$ (Supplementary Fig. 1A). Loss of A1 did not cause a survival defect in GM-CSFstimulated neutrophils. Here, we examined the survival of neutrophils activated with LPS plus GM-CSF from $A 1^{-/-}$, $\mathrm{Mcl}-1^{+/-}$, and $\mathrm{Mcl}-1^{+/-} \mathrm{Al}^{-/-}$mice. Without stimulation, $\mathrm{Mcl}-1^{+/-}$neutrophils had a significant survival disadvantage compared to their wild-type and $A 1^{-/-}$counterparts and no further decrease in cell survival was observed in $\mathrm{Mcl}-1^{+/-} \mathrm{Al}^{-/-}$neutrophils (Fig. 1a). Presumably, this increased apoptosis observed in $\mathrm{Mcl}-1^{+/-}$ neutrophils is due to the in vitro conditions, as we saw normal neutrophil numbers in vivo in ${\mathrm{Mcl}-1^{+/-}}$or $\mathrm{Mcl}$ $1^{+/-} A 1^{-/-}$mice (Supplementary Fig. 1B). After activation

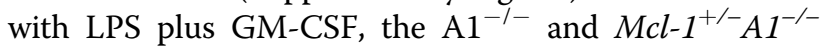

\footnotetext{
Correspondence: Marco J. Herold (herold@wehi.edu.au)

${ }^{1}$ The Walter and Eliza Hall Institute of Medical Research, Parkville, VIC, Australia ${ }^{2}$ Department of Medical Biology, University of Melbourne, Parkville, VIC, Australia

Full list of author information is available at the end of the article
}

neutrophils exhibited significantly poorer survival, whilst Mcl-1 $1^{+/-}$neutrophils behaved similarly to wild-type cells (Fig. 1b). LPS treatment alone was ineffective at promoting a survival advantage and failed to induce neutrophil blasting or upregulate pro-survival MCL-1 expression (Supplementary Fig. 2A-C). GM-CSF treatment alone promoted survival, blasting, and MCL-1 upregulation in wild-type and $A 1^{-/-}$cells $^{3}$. GM-CSF is known to induce expression of the TLR4 co-receptor CD14. We observed marked upregulation of CD14 on neutrophils after GM-CSF stimulation, and more so after treatment with GM-CSF plus LPS (Supplementary Fig. $2 C)$. Hence, the survival defect of LPS plus GM-CSFstimulated $A 1^{-1-}$ neutrophils could be due to a lack of increased A1 expression, contributing to the survival of activated neutrophils ${ }^{8,9}$.

Neutrophils are highly sensitive to FAS-induced apoptosis ${ }^{1}$, but this death is delayed when they are activated by LPS plus GM-CSF ${ }^{1}$. We analyzed FASL-induced apoptosis with and without LPS plus GM-CSF stimulation in neutrophils from $\mathrm{Al}$ and $\mathrm{Mcl}-1$ mutant mice. Additionally, FASL-induced apoptosis in neutrophils is dependent on caspase-8-mediated activation of the pro-apoptotic BCL-2 family member BID (called tBID) ${ }^{10}$, which A1 binds to with high affinity ${ }^{11}$. We therefore also included $\mathrm{Bid}^{-/-}$mice ${ }^{12}$ as a control in our experiments and, furthermore, generated $\mathrm{Bid}^{-/-} \mathrm{A} 1^{-/-}$mice in order to examine whether any effects seen in the $A 1^{-/-}$cells were dependent on A1-tBID interactions.

$\mathrm{Mcl}-1^{+/-}$(and $\mathrm{Mcl}-1^{+/-} \mathrm{Al}^{-/-}$) neutrophils died quicker than wild-type cells after FASL treatment (Fig. 1c). FASLinduced apoptosis was greater than basal apoptosis in culture (Supplementary Fig. 3). Bid ${ }^{-/-}$neutrophils were protected from FASL-induced apoptosis ${ }^{10}$. LPS plus GM- 

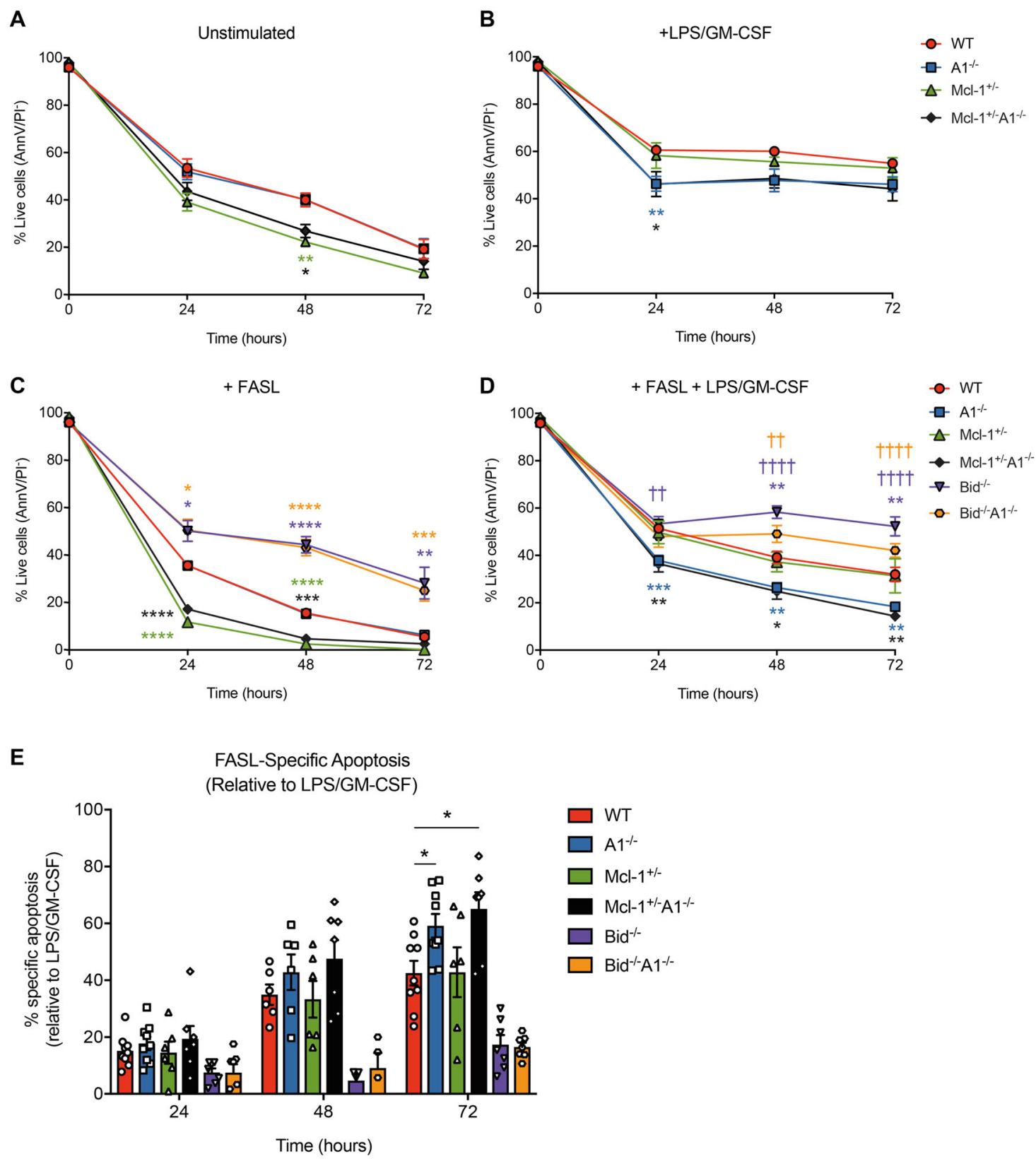

Fig. 1 Survival analysis of neutrophils from mice with the indicated genotypes cultured in a simple medium (no added cytokines), $\mathbf{b}$ after stimulation with $10 \mathrm{ng} / \mathrm{mL}$ GM-CSF plus $10 \mathrm{ng} / \mathrm{mL}$ LPS, c after treatment with Fc-FASL $(0.6 \mathrm{ng} / \mathrm{mL})$, and $\mathbf{d}$ after stimulation with LPS plus GM-CSF (10 ng/mL each) and Fc-FASL $(0.6 \mathrm{ng} / \mathrm{mL})$. e FASL-specific apoptosis when compared to survival of cells stimulated with LPS plus GM-CSF. Data are from five

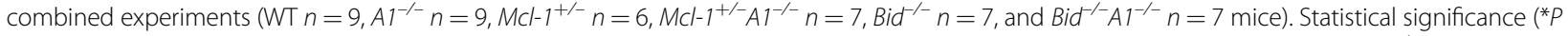
$\left.<0.05,{ }^{* *} P<0.01,{ }^{* * *} P<0.001,{ }^{* * *} P<0.0001\right)$ was determined using Student's $t$-test at each timepoint compared to $W T\left(^{*}\right)$ or $A 1^{-/-}(\dagger)$.

CSF protected both wild-type and $\mathrm{Mcl}-1^{+/-}$neutrophils against FASL-induced killing (Fig. 1d). In contrast, $A 1^{-/-}$ and $\mathrm{Mcl}-\mathrm{1}^{+/-} \mathrm{A1}^{-/-}$neutrophils exhibited significantly more apoptosis across all time points after treatment with FASL in LPS plus GM-CSF-activated neutrophils. Taking into account the increase in apoptosis after LPS plus GMCSF stimulation in $A 1^{-/-}$neutrophils. We observed a trend towards more FASL-specific apoptosis in the A1- deficient cells, although this only reached statistical significance at $72 \mathrm{~h}$ (Fig. 1e). The amount of FASL-specific apoptosis did not differ between $\mathrm{Bid}^{-/-}$and $\mathrm{Bid}^{-/-} \mathrm{Al}^{-/-}$ cells, indicating that the increased sensitivity of activated $A 1^{-/-}$neutrophils to FASL killing is mediated by tBID. $\mathrm{Bid}^{-/-} A 1^{-/-}$neutrophils displayed lower viability than their $\mathrm{Bid}^{-/-}$counterparts, both after LPS plus GM-CSF stimulation (Supplementary Fig. 4) and with the 
combination of LPS, GM-CSF, and FASL (Fig. 1d), fitting with the role we showed for A1 in promoting cell survival after LPS plus GM-CSF stimulation alone.

Collectively, we demonstrate that upregulation of A1 after stimulation imparts a survival advantage in neutrophils, including FASL-induced apoptosis. However, A1's role is relatively small, and other factors must also regulate the survival of activated neutrophils. These results suggest a previously unrecognized role for A1 in promoting neutrophil survival in an inflammatory context.

\section{Acknowledgements}

We acknowledge the invaluable contributions of the animal caretaker staff at the Walter \& Eliza Hall Institute for animal husbandry, namely Giovanni Siciliano, Krystal Hughes and Daniel Fayle. We also acknowledge the flow cytometry facilities of the Walter \& Eliza Hall Institute, led by Simon Monard and his team. This work was supported by grants and fellowships from the Australian National Health and Medical Research Council (NHMRC) (Project Grants 1186575 and 1145728 to M.J.H., 1143105 to M.J.H. and A.S., 1159658 to M.J.H., Program Grant 1016701 to A.S. and Fellowships 1020363 to A.S., 1156095 to M.J.H.), the Leukemia and Lymphoma Society of America (LLS SCOR 7001-13 to A.S. and M.J.H.), the Cancer Council of Victoria (project grant 1147328 to M.J.H., 1052309 to A.S., and Venture Grant to M.J.H. and A.S.), as well as by operational infrastructure grants through the Australian Government Independent Research Institute Infrastructure Support Scheme (361646 and 9000220) and the Victorian State Government Operational Infrastructure Support Program.

\section{Author details}

The Walter and Eliza Hall Institute of Medical Research, Parkville, VIC, Australia. 2Department of Medical Biology, University of Melbourne, Parkville, VIC, Australia. ${ }^{3}$ Centre for Innate Immunity and Infectious Diseases, Hudson Institute of Medical Research, Clayton, VIC, Australia. ${ }^{4}$ Department of Molecular and Translational Science, Monash University, Clayton, VIC, Australia. ${ }^{5}$ Present address: Research Institute of Molecular Pathology, Vienna Biocenter, Vienna, Austria

\section{Author contributions}

R.L.S. performed and designed most experiments and wrote the manuscript; L. G. helped to perform experiments and write the manuscript; K.E.L. helped with discussions and advice on neutrophil experiments and write the manuscript; L. A.O. provided reagents and helped with advice on FASL experiments and write the manuscript; A.S. and M.J.H. planned the project, were involved in experimental design and helped to write the manuscript.
Conflict of interest

The authors declare that they have no conflict of interest.

\section{Publisher's note}

Springer Nature remains neutral with regard to jurisdictional claims in published maps and institutional affiliations.

Supplementary Information accompanies this paper at (https://doi.org/ 10.1038/s41419-020-2676-9).

Received: 4 June 2020 Accepted: 8 June 2020

Published online: 18 June 2020

\section{References}

1. O'Donnell, J. A. et al. Fas regulates neutrophil lifespan during viral and bacterial infection. J. Leukoc. Biol. 97, 321-326 (2015).

2. Strasser, A., Cory, S. \& Adams, J. M. Deciphering the rules of programmed cell death to improve therapy of cancer and other diseases. EMBO J. 30, 3667-3683 (2011).

3. Schenk, R. L. et al. Characterisation of mice lacking all functional isoforms of the pro-survival $\mathrm{BCL}-2$ family member $\mathrm{A} 1$ reveals minor defects in the haematopoietic compartment. Cell Death Differ. 24, 534-545 (2017).

4. Dzhagalov, I., John, A. S. \& He, Y.-W. The antiapoptotic protein Mcl-1 is essential for the survival of neutrophils but not macrophages. Blood 109, 1620-1626 (2007).

5. Csepregi, J. Z. et al. Myeloid-specific deletion of Mcl-1 yields severely neutropenic mice that survive and breed in homozygous form. J. Immunol. 201, 3793-3803 (2018)

6. Brinkmann, K. et al. The combination of reduced MCL-1 and standard chemotherapeutics is tolerable in mice. Cell Death Differ. 24, 2032-2043 (2017).

7. Kurt-Jones, E. A. et al. Role of Toll-like receptor 2 (TLR2) in neutrophil activation: GM-CSF enhances TLR2 expression and TLR2-mediated interleukin 8 responses in neutrophils. Blood 100, 1860-1868 (2002).

8. Vier, J., Groth, M., Sochalska, M. \& Kirschnek, S. The anti-apoptotic Bcl-2 family protein A1/Bfl-1 regulates neutrophil survival and homeostasis and is controlled via PI3K and JAKSSTAT signaling. Cell Death Dis. 7, e2103 (2016).

9. Renshaw, S. A. et al. Inflammatory neutrophils retain susceptibility to apoptosis mediated via the Fas death receptor. J. Leukoc. Biol. 67, 662-668 (2000).

10. Wicki, S. et al. Loss of BID delays FASL-induced cell death of mouse neutrophils and aggravates DSS-induced weight loss. Int. J. Mol. Sci. 19, 684 (2018).

11. Werner, A. B., Vries, E., de, Tait, S. W. G., Bontjer, I. \& Borst, J. Bcl-2 family member Bfl-1/A1 sequesters truncated bid to inhibit its collaboration with proapoptotic Bak or Bax. J. Biol. Chem. 277, 22781-22788 (2002).

12. Kaufmann, T. et al. The BH3-only protein bid is dispensable for DNA damageand replicative stress-induced apoptosis or cell-cycle arrest. Cell 129, 423-433 (2007). 